\title{
The application of high-throughput sequencing technology to analysis of $a m o A$ phylogeny and environmental niche specialisation of terrestrial bacterial ammonia-oxidisers
}

\author{
Axel Aigle, James I. Prosser and Cécile Gubry-Rangin * (1)
}

\begin{abstract}
Background: Characterisation of microbial communities increasingly involves use of high throughput sequencing methods (e.g. MiSeq Illumina) that amplify relatively short sequences of 16S rRNA or functional genes, the latter including ammonia monooxygenase subunit $\mathrm{A}(\mathrm{a} m \circ \mathrm{A})$, a key functional gene for ammonia oxidising bacteria (AOB) and archaea (AOA). The availability of these techniques, in combination with developments in phylogenetic methodology, provides the potential for better analysis of microbial niche specialisation. This study aimed to develop an approach for sequencing of bacterial and archaeal amoA genes amplified from soil using bioinformatics pipelines developed for general analysis of functional genes and employed sequence data to reassess phylogeny and niche specialisation in terrestrial bacterial ammonia oxidisers.

Results: amoA richness and community composition differed with bioinformatics approaches used but analysis of MiSeq sequences was reliable for both archaeal and bacterial amoA genes and was used for subsequent assessment of potential niche specialisation of soil bacteria ammonia oxidisers. Prior to ecological analysis, phylogenetic analysis of Nitrosospira, which dominates soil $A O B$, was revisited using a phylogenetic analysis of $16 \mathrm{~S}$ rRNA and amoA genes in available AOB genomes. This analysis supported congruence between phylogenies of the two genes and increased previous phylogenetic resolution, providing support for additional gene clusters of potential ecological significance. Analysis of environmental sequences using these new sequencing, bioinformatics and phylogenetic approaches demonstrated, for the first time, similar niche specialisation in AOB to that in $\mathrm{AOA}$, indicating $\mathrm{pH}$ as a key ecological factor controlling the composition of soil ammonia oxidiser communities.
\end{abstract}

Conclusions: This study presents the first bioinformatics pipeline for optimal analysis of Illumina MiSeq sequencing of a functional gene and is adaptable to any amplicon size (even genes larger than $500 \mathrm{bp}$ ). The pipeline was used to provide an up-to-date phylogenetic analysis of terrestrial betaproteobacterial $a m \circ A$ genes and to demonstrate the importance of soil pH for their niche specialisation and is broadly applicable to other ecosystems and diverse microbiomes.

Keywords: Nitrification, amoA, $16 \mathrm{~S}$ rRNA, Archaea, Bacteria, Illumina MiSeq, pH

\footnotetext{
* Correspondence: c.rangin@abdn.ac.uk

School of Biological Sciences, Cruickshank Building, University of Aberdeen,

St. Machar Drive, Aberdeen AB24 3UU, UK
}

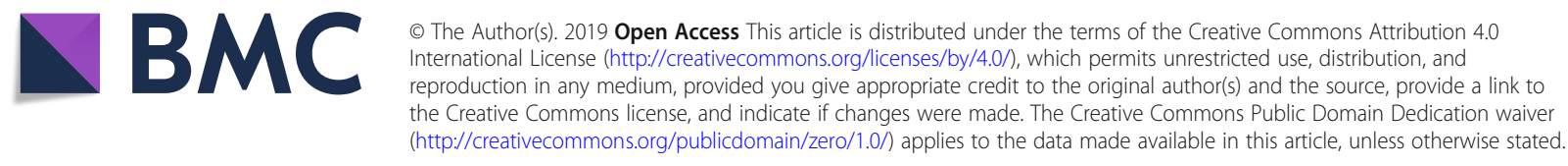




\section{Background}

Despite advances in our understanding of niche specialisation of ammonia oxidisers during the past 20 years, the successive discoveries of archaeal ammonia oxidisers (AOA) [1] and complete ammonia oxidisers (comammox) $[2,3]$ have focussed recent research activities on these organisms. In particular, in terrestrial environments, $\mathrm{pH}$ has been described as the most important soil factor regulating the AOA ecological and evolutionary adaptation $[4,5]$ and, while there is currently insufficient information on which to assess comammox growth or adaptation in this environment, the ecophysiology of soil comammox appears to differ from that of strains that have been cultivated and described to date [6]. In contrast, the modern technological and methodological advances have not benefited analysis of the longerknown bacterial ammonia oxidisers (AOB), despite demonstration of their significant role in nitrification, particularly in managed and heavily fertilised agricultural soils [7-9]. In particular, phylogenetic analyses have gained in complexity and consequent accuracy, and finer phylogenetic analyses of soil AOB [10-14] would benefit from increases in knowledge of the diversity of soil $\mathrm{AOB}$ since previous in-depth studies (e.g. [15]). In particular, reassessment of the terrestrial Nitrosospira phylogeny is required using more advanced phylogenetic methods, as this genus dominates soil $\mathrm{AOB}$ communities.

Increased phylogenetic resolution improves the ability to assess microbial niche specialisation, at least in organisms whose phylogenetic structure reflects their ecological niche [16]. Several environmental factors have been proposed as important for controlling the community structure of terrestrial $\mathrm{AOB}$, including the nature and quantity of organic matter [17], mean annual temperature [18], amount of nitrogen fertilisation [19] and soil $\mathrm{pH}$ [20]. However, understanding of the niche differentiation of these microorganisms in natural unfertilised environments remains limited and the role of terrestrial Nitrosospira in nitrous oxide emissions [8,9] justifies the need for an improved analysis of their niche specialisation to clarify their ecological adaptation and environmental impact. This analysis would specifically test the extent to which $\mathrm{pH}$ is a key factor controlling the ecological distribution of AOB in soils, as previously demonstrated for AOA in natural environments $[4,5]$, by using a soil $\mathrm{pH}$ gradient of several unfertilised land management (grassland, forest, agricultural or moorland) soils.

Many studies target the 16S rRNA gene to characterise phylogenetic diversity within a sample, while others target genes involved in specific ecosystem functions, facilitating ecological and evolutionary predictions within a functional group. The ammonia monooxygenase subunit A $(a m o A)$ gene targets an ecosystem function, ammonia oxidation, which is the rate-limiting step in nitrification, a key process in biogeochemical cycling of nitrogen. The amo $A$ gene has been extensively used to estimate the abundance and diversity of bacterial (AOB) and archaeal ammonia oxidisers (AOA) and has provided evidence for their high phylogenetic diversity in natural environments, including soil [21]. Therefore, the amoA gene was chosen as the optimal functional gene to analyse soil $\mathrm{AOB}$ diversity and two sets of primers have been mainly employed in terrestrial environments, amoA1F/amoA-2R [22] and CrenamoA23f/CrenamoA616r [23], amplifying 429-bp and 629-bp fragments of bacterial and archaeal $a m o A$ genes, respectively. Developments in high-throughput sequencing (HTS) technologies have greatly increased our ability to characterise natural microbial communities, through significant increases in the depth and accuracy of sequencing of genes of interest, amplified from environmental DNA (see [24] for a recent review). Currently, one of the most commonly applied approaches is short-read sequencing (e.g. Illumina) technology, producing a high number of sequences ( $>15 \mathrm{Gbp}$ per Illumina MiSeq V3 run) with high accuracy (99.9\% at QC30) but short sequence length (<500 bp). Surprisingly, this approach has not been applied to terrestrial ammonia oxidisers using the above primers [21, 22], probably due to the large size of the amplicons (mainly for the AOA). Therefore, this study provided the opportunity to develop a bioinformatics pipeline for amplicons of various size (including those $>500$ bp) and its validity was assessed by comparison with a previously acquired 454 sequencing dataset with known phylogenetic resolution [4].

The aims of this study were, therefore, i) to revisit the amoA phylogeny of terrestrial Nitrosospira and improve its phylogenetic resolution; ii) to improve the analysis of niche specialisation of terrestrial Nitrosospira to clarify their ecological adaptation and test the extent to which $\mathrm{pH}$ is a key factor in their ecological distribution; and iii) to provide an Illumina MiSeq V3 sequencing approach for environmental analysis of the ammonia monooxygenase subunit A $(a m o A)$ gene present in both bacterial and archaeal ammonia oxidisers, applicable to other functional genes.

\section{Results and Discussion}

The aims of the study were addressed by performing archaeal and bacterial amoA gene Illumina MiSeq sequencing, respectively, on 47 and 33 UK soils for which environmental data were available. In addition, $16 \mathrm{~S}$ rRNA and $a m o A$ genes retrieved from 56 available Nitrosospira strains were used for phylogenetic reconstructions and assessment of phylogenetic congruency between them. 


\section{Nitrosospira classification}

Two genera of betaproteobacterial ammonia oxidisers have been described, Nitrosomonas and Nitrosospira [25], the latter including two previously described genera, Nitrosolobus [26] and Nitrosovibrio [27]. This study focused on the Nitrosospira genus, which dominates betaproteobacterial ammonia oxidiser communities in soil. The 16S rRNA gene sequences from cultivated Nitrosospira isolates and those amplified from environmental samples have previously been classified within seven lineages, for which phylogenetic node support was not high [10-14]. Sequences of six of these lineages were retrieved in the present study at a high taxonomic ranking (sub-clades) using 56 available Nitrosospira strains (Fig. 1 and Additional file 1: Figure S1), the exception being cluster 1 [10], for which no cultured isolate has yet been obtained. Analysis of $a m o A$ and $16 \mathrm{~S}$ rRNA gene sequences of these Nitrosospira strains, employing a Maximum-Likelihood phylogenetic framework, delineated 17 and 19 phylogenetic clusters, respectively (Fig. 1 and Additional file 1: Figure S1, Table 1), with most of the diversity being within the previously defined cluster 3. The majority of the $56 \mathrm{AOB}$ strains analysed here contained a single amoA gene copy (Additional file 1: Table S1), but several Nitrosospira spp. genomes contain $>1$ different but highly homologous amo $A$ genes, which probably originated from duplication events rather than horizontal gene transfer [30-32]. This finding, along with the presence of $>1$ amoA gene copy in most described Nitrosomonas genomes [33], has important consequences for quantification of terrestrial $\mathrm{AOB}$ in environmental communities using quantitative $\mathrm{PCR}$ analysis of $a m o A$ genes. Most nodes at the roots of individual clusters in both phylogenetic trees were strongly supported (>80\%), while support for more ancestral phylogenetic branching was supported for most of the nodes in both the 16S rRNA gene and the amoA gene phylogenies, even if some paraphyletic branching could not be resolved (Fig. 1 and Additional file 1: Figure S1).

The present phylogenetic approach demonstrates that the previous $16 \mathrm{~S}$ rRNA gene phylogenetic clustering for lineage 3 requires future refinement, as this previously described cluster is paraphyletic and contains numerous distinct clusters. While the present phylogenetic analysis did not aim to represent the extent of Nitrosospira diversity (on neither 16S rRNA or amoA genes), it provides evidence for the existence of clear and distinct lineages using both marker genes of cultivated strains (Fig. 1; Table 1 and Additional file 1: Table S1) and, in contrast to earlier analysis [34], demonstrated a strong phylogenetic congruence between $a m o A$ and 16 rRNA genes using a tanglegram approach (Fig. 1 and Additional file 1: Figure S2).

\section{Niche specialisation of terrestrial bacterial ammonia oxidisers}

A large majority (99.98\% of the 199,295 sequences) of the environmental sequences obtained from the 33 soils amplified using bacterial primers affiliated to the Nitrosospira genus, which has been described as the most abundant AOB genus in unfertilised soils, while Nitrosomonas phylotypes are more frequently retrieved from fertilised or ammonia-rich environments [30]. These $a m o A$ sequences were affiliated to ecologically coherent
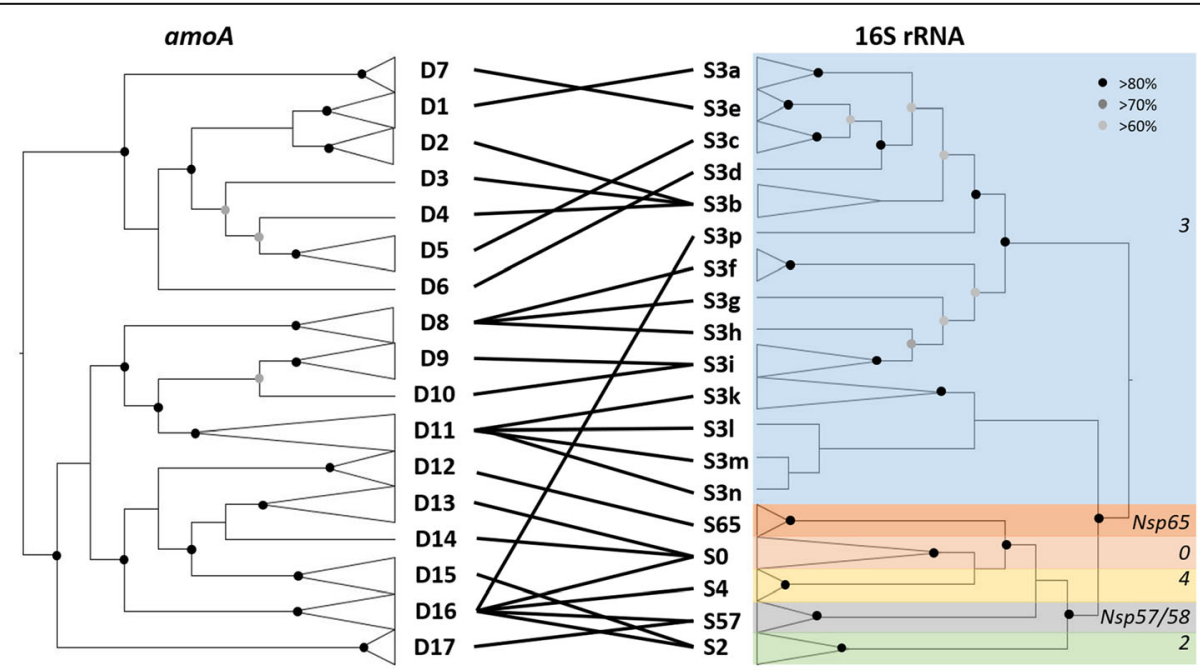

Fig. 1 Congruence between amoA and 16S rRNA gene cladograms for Nitrosospira cultures and genomes used in this study. Lines represent correspondence between clusters of the two phylogenies. Names of the phylogenetic clusters indicated in bold on the two phylogenetic trees are arbitrary, while those in italics correspond to previously published 165 rRNA phylogenies $[28,29]$. Details of the strain identity within each cluster are given in Table 1 and node bootstrap values are indicated by circles with different shadings 
Table 1 Terrestrial Nitrosospira AOB strains used in this study with related $a m o A$ and 165 rRNA gene phylogenetic affiliations (based on the phylogenetic trees presented in Fig. 1)

\begin{tabular}{|c|c|c|}
\hline Organism & $\begin{array}{l}\text { amoA gene } \\
\text { lineage }\end{array}$ & $\begin{array}{l}\text { 16S rRNA gene } \\
\text { lineage }\end{array}$ \\
\hline Nitrosospira multiformis Nsp16 & D1 & S3a \\
\hline Nitrosospira sp. Nsp18 & D1 & S3a \\
\hline Nitrosospira sp. 1 Nsp11 & D1 & S3a \\
\hline Nitrosospira briensis C-128 & D2 & $\mathrm{S3b}$ \\
\hline Nitrosospira sp. Nsp1 & D2 & $\mathrm{S} 3 \mathrm{~b}$ \\
\hline Nitrosospira sp. NRS527 & D2 & $\mathrm{S} 3 \mathrm{~b}$ \\
\hline Nitrosospira sp. Nsp40 & D3 & S3b \\
\hline Nitrosospira sp. Nsp22 & D4 & S3b \\
\hline Nitrosospira tenuis Nv12 & D5 & S3c \\
\hline Nitrosospira sp. Nv6 & D5 & $\mathrm{S} 3 \mathrm{c}$ \\
\hline Nitrosospira sp. Nsp37 & D5 & S3c \\
\hline Nitrosospira sp. Nv4 & D5 & S3c \\
\hline Nitrosospira sp. Nsp62 & D6 & S3d \\
\hline Nitrosospira briensis Nsp10 & D7 & S3e \\
\hline Nitrosospira briensis Nsp8 & D7 & S3e \\
\hline Nitrosospira sp. Nsp14 & D8 & S3f \\
\hline Nitrosospira sp. Nsp17 & D8 & S3f \\
\hline Nitrosospira sp. Nsp2 & D8 & $\mathrm{S3g}$ \\
\hline Nitrosospira sp. Nsp44 & D8 & $\mathrm{S} 3 \mathrm{~h}$ \\
\hline Nitrosospira multiformis $24 \mathrm{C}$ & D9 & S3i \\
\hline Nitrosospira sp. L115 & D9 & S3i \\
\hline Nitrosospira sp. A16 & D9 & S3i \\
\hline Nitrosospira sp. AF & D9 & S3i \\
\hline Nitrosospira tenuis Nv1 & D10 & S3i \\
\hline Nitrosospira multiformis ATCC 25196 & D11 & S3k \\
\hline Nitrosospira multiformis NI13 & D11 & S3k \\
\hline Nitrosospira multiformis NI4 & D11 & S3k \\
\hline Nitrosospira multiformis NI18 & D11 & S3k \\
\hline Nitrosospira multiformis NI15 & D11 & S3k \\
\hline Nitrosospira multiformis NI14 & D11 & S3k \\
\hline Nitrosospira multiformis NI7 & D11 & S3k \\
\hline Nitrosospira multiformis NI8 & D11 & S3k \\
\hline Nitrosospira multiformis NI12 & D11 & S31 \\
\hline Nitrosospira multiformis NI2 & D11 & $\mathrm{S} 3 \mathrm{~m}$ \\
\hline Nitrosospira multiformis NI3 & D11 & S3n \\
\hline Nitrosospira sp. Nsp65 & D12 & S65 \\
\hline Nitrosospira sp. 56-18 & D12 & S65 \\
\hline Nitrosospira sp. Nsp5 & D13 & so \\
\hline Nitrosospira multiformis NI1 & D13 & SO \\
\hline Nitrosospira sp. Nsp13 & D13 & SO \\
\hline Nitrosospira sp. Nsp6 & D13 & so \\
\hline Nitrosospira sp. Nsp12 & D13 & so \\
\hline
\end{tabular}

Table 1 Terrestrial Nitrosospira AOB strains used in this study with related $a m o A$ and 165 rRNA gene phylogenetic affiliations (based on the phylogenetic trees presented in Fig. 1)

\begin{tabular}{lll} 
(Continued) & $\begin{array}{l}\text { amoA gene } \\
\text { lineage }\end{array}$ & $\begin{array}{l}16 \mathrm{~S} \text { rRNA gene } \\
\text { lineage }\end{array}$ \\
\hline Nitrosospira sp. 40KI & $\mathrm{D} 13$ & $\mathrm{~S} 0$ \\
Nitrosospira sp. NpAV & $\mathrm{D} 14$ & $\mathrm{~S} 0$ \\
Nitrosospira lacus APG3 & $\mathrm{D} 16$ & $\mathrm{~S} 0$ \\
Nitrosospira sp. Ka4 & $\mathrm{D} 16$ & $\mathrm{~S} 4$ \\
Nitrosospira sp. Ka3 & $\mathrm{D} 16$ & $\mathrm{~S} 4$ \\
Nitrosospira sp. Nsp41 & $\mathrm{D} 16$ & $\mathrm{~S} 3 \mathrm{p}$ \\
Nitrosospira sp. Nsp58 & $\mathrm{D} 16$ & $\mathrm{~S} 57$ \\
Nitrosospira sp. B6 & $\mathrm{D} 16$ & $\mathrm{~S} 2$ \\
Nitrosospira sp. III7 & $\mathrm{D} 15$ & $\mathrm{~S} 2$ \\
Nitrosospira sp. O13 & $\mathrm{D} 15$ & $\mathrm{~S} 2$ \\
Nitrosospira sp. O4 & $\mathrm{D} 15$ & $\mathrm{~S} 2$ \\
Nitrosospira sp. AHB1 & $\mathrm{D} 15$ & $\mathrm{~S} 2$ \\
Nitrosospira sp. Nsp57 & $\mathrm{D} 17$ & $\mathrm{~S} 57$ \\
Nitrosospira sp. N15 & $\mathrm{D} 17$ & $\mathrm{~S} 57$
\end{tabular}

phylogenetic AOB clusters (Fig. 2a) and multivariate statistical analysis of their ecological distribution demonstrated greatest correlations with soil $\mathrm{pH}$ and, to a lesser extent, $\mathrm{C}: \mathrm{N}$ ratio across a range of phylogenetic scales (Table 2, see Additional file 1: Table S2 for detailed results). There is strong evidence for $\mathrm{pH}$ specialisation of AOA [4], but this strong influence of $\mathrm{pH}$ on $\mathrm{AOB}$ phylotype distribution over multiple phylogenetic scales has not been described previously, suggesting a role for $\mathrm{pH}$ in determining community composition of all terrestrial ammonia oxidisers. A heatmap representing the relative abundance of each phylogenetic cluster in each soil indicated higher relative abundance of four clusters (D8, D15, D16 and D19) (Fig. 2b) and dominance of these clusters strongly influenced the general $\mathrm{pH}$ association with overall community similarity pattern. Sequences affiliating to the cluster D8 were particularly abundant in neutro-alkaline soils. In contrast to such classical AOB ecological niche distribution, most $\mathrm{AOB}$ sequences in neutro-acidic soils affiliated with the D15, D16 and D19 clusters, but more genomes and cultures representative of these clusters are required for characterisation of their phenotypes. For example, AOB ureolytic activity has been proposed, among others, as a growth strategy in acidic conditions [21]. Other less abundant phylogenetic clusters would also benefit from further genomic and cultivation efforts (see Fig. 2a).

\section{Comparison of sequencing technologies}

Comparisons of the diversity of environmental amo $A$ gene sequences were made using both OTU richness 


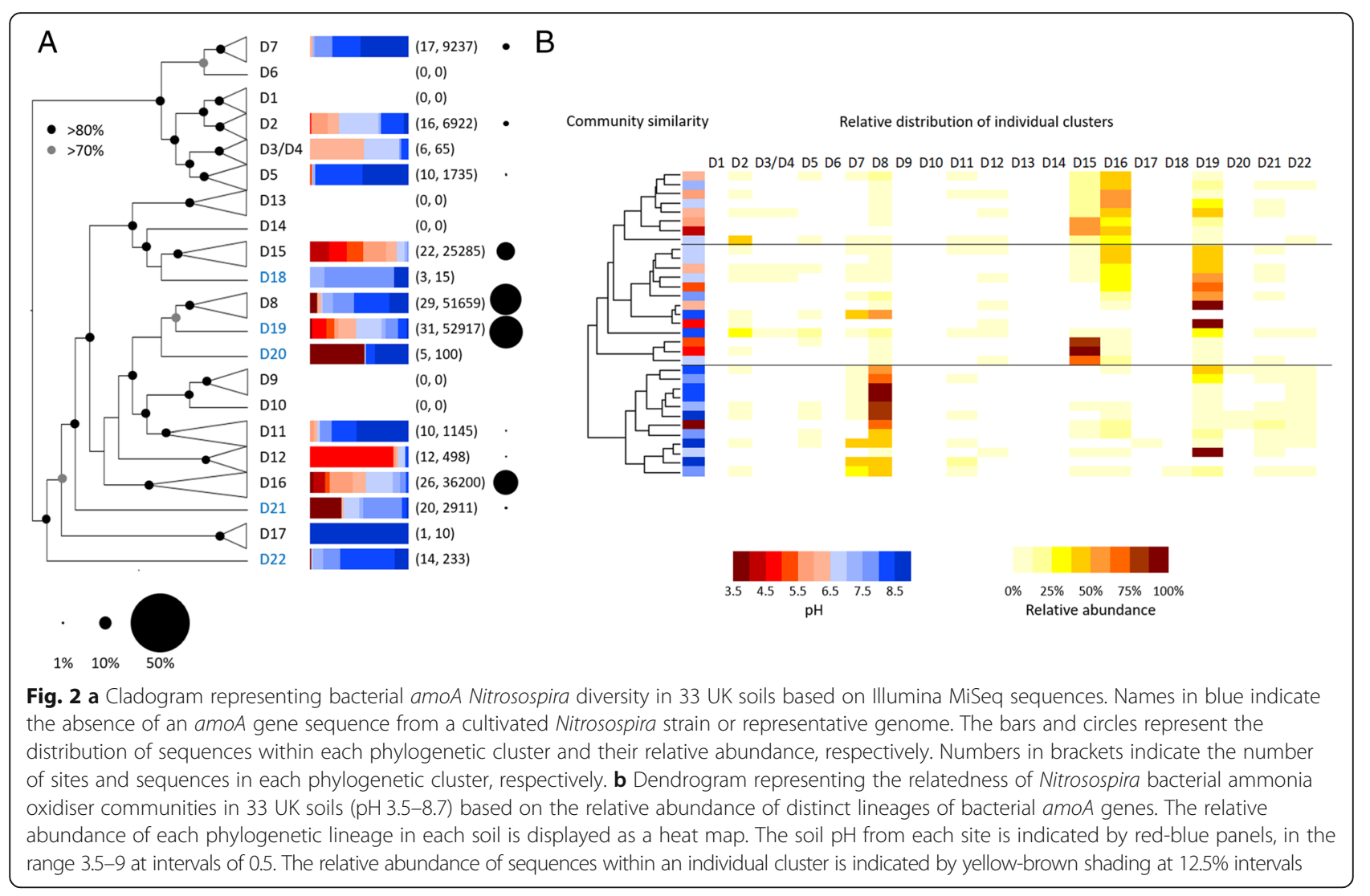

(at $100 \%$ sequence similarity) and microbial community composition for archaea and bacteria independently using the different sequencing approaches, 454 (only available for AOA and previously described in [4]) and Illumina MiSeq sequencing. Both measures varied with sequencing technology and associated bioinformatics pipeline (Fig. 3; Additional file 1: Table S3). Despite a low error rate for Illumina sequencing, the read length $(2 \times 300 \mathrm{bp})$ limited the number of cleaned sequences (Additional file 1: Table S3). Two bioinformatics pipelines ('assembly' vs. 'gap') were used to analyse the AOB amoA amplicons (490-bp), due to overlapping of the paired-end sequences. Rarefied richness was similar or higher for the 'gap' pipeline than the 'assembly' pipeline, due to the higher restrictive size selection in the latter (Additional file 1: Table S3). Despite detection of similar to greater richness, phylogenetic assignment of sequences was differentially affected by the pipeline used (Bray-Curtis $\geq 0.3$ for 4 soils; Fig. $3 b$ ). Community dissimilarity obtained using the full-length 'assembly' and 'assembly-gapped' sequences indicated that the 'gapped' region contains important phylogenetic information, especially for the sequences present in acidoneutral soils (Fig. 3b). However, a similar comparative analysis of AOA amoA sequences, using previously obtained 454 sequences (i.e. 454 full-length sequences vs 454-gapped sequences), indicated that deletion of the central archaeal $a m o A$ gene region did not impact significantly on estimated archaeal community composition (Bray-Curtis $=0$ for all 7 soils; Fig. 3a) or phylogenetic reconstruction (Additional file 1: Figure S4; Euclidian distance between the 2 trees $=0.28$ ). The difference in community composition between the AOA 454 and the AOA MiSeq 'gap' (Fig. 3a) certainly derives from different sampling dates for the 2 different technologies. Therefore, these analyses were used to validate the MiSeq 'gap' and 'assembly' bioinformatics pipelines for AOA and $\mathrm{AOB} a m o A$ sequencing, respectively). These findings suggest that Illumina MiSeq sequencing can be successfully used to provide a good characterisation of $\mathrm{AOA}$ and $\mathrm{AOB}$ amoA amplicon sequences to infer their community structure. The sequencing bioinformatics pipelines presented here are freely available on GitHub (https://github.com/AigleAxel/ amoA_MiSeq_sequencing/) allowing their implementation for other functional genes of interest. Specific advantages and associated limitations of each bioinformatics pipeline (e.g. high recovered diversity for the 'gap' pipeline and high confidence of sequence phylogenetic affiliation for the 'assembly' pipeline) indicate the requirement for thorough comparison of approaches for cleaning of sequencing data for any novel analysed gene. 
Table 2 Correlations between measured environmental factors and relative abundance of bacterial ammonia oxidiser lineages (identified at different identity thresholds) in 33 soils used for analysis of bacterial amoA gene sequences

\begin{tabular}{lllll}
\hline Environmental & \multicolumn{4}{l}{ Identity (\%) } \\
\cline { 2 - 5 } factors & 90 & 95 & 97 & 100 \\
\hline $\mathrm{pH}$ & $0.001^{* * *}$ & $0.001^{* * *}$ & $0.001^{* * *}$ & $0.001^{* * *}$ \\
$\mathrm{C}$ & 0.274 & $0.003^{* *}$ & $0.006^{* *}$ & $0.001^{* * *}$ \\
$\mathrm{~N}$ & 0.166 & $0.016^{*}$ & $0.020^{*}$ & $0.001^{* * *}$ \\
$\mathrm{C}: \mathrm{N}$ & $0.001^{* * *}$ & $0.002^{* *}$ & $0.003^{* *}$ & $0.002^{* *}$ \\
Moisture & 0.459 & 0.264 & 0.112 & $0.015^{*}$ \\
LOI & 0.139 & 0.198 & $0.032^{*}$ & 0.072. \\
Vegetation & 0.406 & 0.172 & 0.083. & 0.106 \\
Number of clusters & 14 & 71 & 187 & 3868 \\
\hline Significance codes: $p<0.0011^{* * *} p<0.01 * * p<0.05^{*}$ &
\end{tabular}

Significance codes: $p<0.001 * * ; p<0.01 * * ; p<0.05 *$

\section{Conclusions}

This study offers an optimal bioinformatics pipeline for high-throughput sequencing of functional genes, adaptable to any amplicon size and freely available on GitHub (https://github.com/AigleAxel/amoA_MiSeq_sequencing/).
This tool will be useful to the researchers interested in diverse microbiome communities, especially those working on functional genes of interest larger than 500 bp. The developed pipeline was used to provide a revisited and up-to-date phylogenetic analysis of terrestrial betaproteobacterial ammonia oxidiser $a m o A$ genes and further analysis provided evidence for soil $\mathrm{pH}$ as a key ecological factor controlling the niche specialisation of those microbes.

\section{Material and methods Sample origin, DNA extraction and amoA amplification}

Bacterial and archaeal $a m o A$ sequences were obtained from 26 and 39 soils, respectively, selected from the UK Countryside Survey (http://www.countrysidesurvey.org. $\mathrm{uk} /$ ) and from 7 soil samples from long-term experimental field plots maintained for more than 60 years at a range of soil pH values (SRUC, Craibstone, Scotland, grid reference NJ872104). Soils were selected to include a wide range of characteristics (Additional file 1: Table S4), including $\mathrm{pH}(3.48-8.55), \mathrm{C}: \mathrm{N}$ ratio $(8.5-22.1)$ and moisture content $(14.2-75.1 \%)$, across several ecosystem

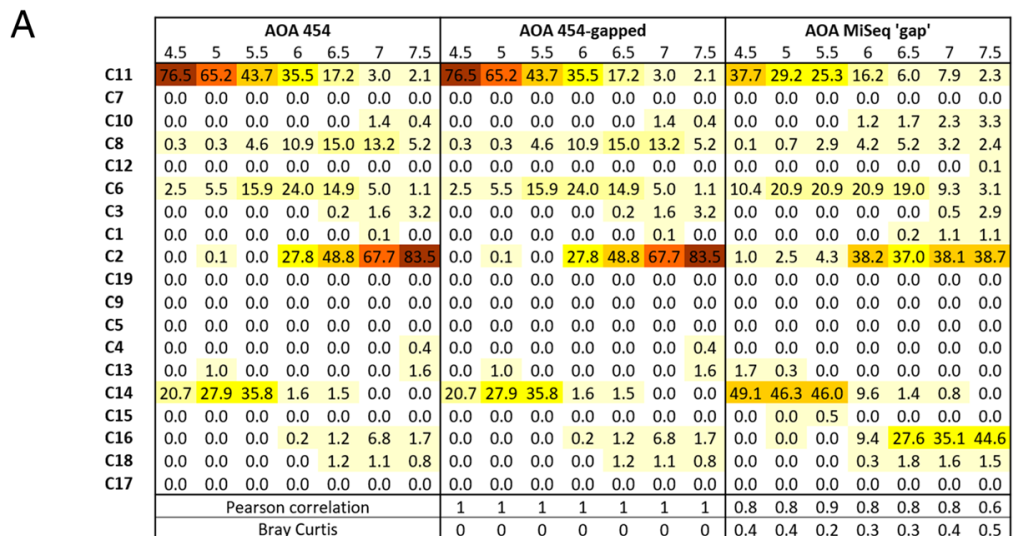

B

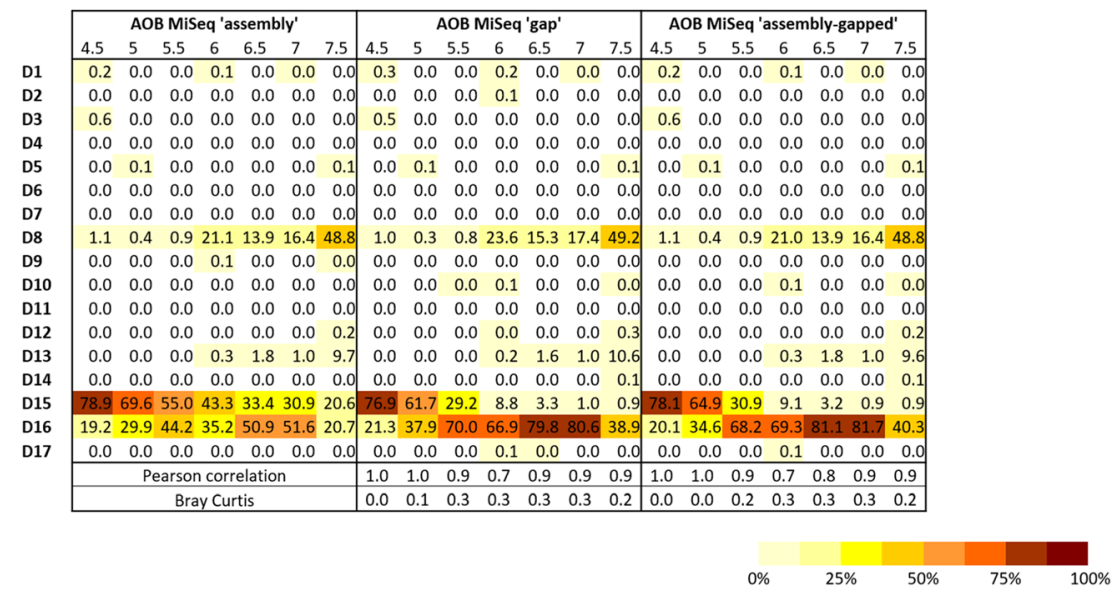

Fig. 3 Heatmap representing the relative abundance of archaeal (a) and bacterial (b) amoA gene sequences within each phylogenetic cluster, produced using different sequencing technologies. The row below each heatmap indicates the Pearson and Bray-Curtis correlations of the microbial community structure for each soil $(\mathrm{pH} 4.5-7.5)$ relative to the technology considered the most accurate 
managements (agricultural, forest, grassland, moorland), and a previous study [4] suggests that they contain the full range of currently known mesophilic terrestrial AOA.

Soil DNA was extracted as described by [35] and modified by [36] and bacterial and archaeal amoA genes were amplified, respectively, using primers amoA-1F/ amoA-2R [22] and CrenamoA23f/CrenamoA616r [23] containing additional specific MiSeq-tailed sequences, following manufacturers' recommendations. All amplifications were performed in a $25-\mu \mathrm{l}$ reaction using the KAPA HiFi HotStart ReadyMix (Kapa Biosystems) with $0.4 \mu \mathrm{M}$ of each primer and $40-60 \mathrm{ng}$ of template. Thermal cycling conditions were $95^{\circ} \mathrm{C}$ for 3 min followed by 35 cycles of $98^{\circ} \mathrm{C}$ for $20 \mathrm{~s}, 58^{\circ} \mathrm{C}$ for $15 \mathrm{~s}, 72^{\circ} \mathrm{C}$ for $15 \mathrm{~s}$ or $20 \mathrm{~s}$ for bacterial and archaeal amoA respectively, followed by $72{ }^{\circ} \mathrm{C}$ for $5 \mathrm{~min}$. Bacterial amoA MiSeq sequencing was performed on all 33 soil samples, allowing the construction of a non-redundant database of representative soil $\mathrm{AOB}$ amoA sequences to complement an equivalent database of AOA amo $A$ sequences obtained using 454 sequencing on 46 soils [4].

\section{High throughput sequencing}

Prior to MiSeq Illumina sequencing, PCR-amplified sequences were cleaned using AMPure ${ }^{\circ} \mathrm{XP}$ beads (Beckman Coulter) and PCR-indexing was performed using the Nextera XT Index Kit according to the manufacturer's protocol. Following further cleaning, library quantification, normalisation and pooling of 144 samples per sequencing run were performed prior to paired-end V3 MiSeq sequencing, enabling production of $2 \times 300$ bp reads.

\section{Sequence analysis bioinformatic pipelines}

The different read lengths of archaeal and bacterial amo $A$ genes generated by Illumina MiSeq sequencing necessitated use of two read assembly strategies. The first, with overlapping reads and termed the 'assembly' pipeline, was used for bacterial amo $A$ only. The second, with non-overlapping reads and termed the 'gap' pipeline, was used for both archaeal and bacterial $a m o A$. For the 'assembly' pipeline, reads were demultiplexed using NextGENe software and, for each sample, paired-end raw reads were trimmed (-- paired) with very low quality filtration (-q 15) using Trim Galore (V0.4.5, [37]) and further filtered using the filterAndTrim command from the DADA2 package $(\operatorname{maxEE}=\mathrm{c}(2,2), \operatorname{maxN}=0)$ [38]. Minimal size selection and read truncation (trun$c$ Len $=c(229,229)$, minLen $=229)$ were applied to allow overlap of 10 bases between the paired-end reads. Assembly was performed using the paired-end assembler PEAR with default parameters [39] and assembled reads were size-selected using usearch (-fastx_truncate -trunclen 448) [40] and subsequently dereplicated at $100 \%$ sequence identity (while keeping the read abundance information) using usearch (-cluster_fast -centroids -sizeout). Finally, dereplicated reads were translated and any read that included a stop codon was deleted prior to removal of chimera and singletons using unoise3 [41].

Initial steps of the 'gap' pipeline were similar to the 'assembly' pipeline except that minimal size selection was modified (-length 200), as a compromise between selection of high-quality reads (especially for reverse reads) and conservation of high-quality information (carried by higher number of nucleotides) to maximise the output number of reads. The reverse reads were reversecomplemented and concatenated with the forward reads (instead of being assembled via PEAR). The following steps (dereplication, amino acid translation, chimera and singleton removal) were the same as in the 'assembly' pipeline.

Comparison of the different sequencing methodologies was performed for the seven Craibstone samples following a blast-assignment of sequences to the different phylogenetic clusters (see [5] and below for AOA and $\mathrm{AOB} a m o A$ databases, respectively) and the proportions of sequences affiliated to each phylogenetic cluster within each soil sample were represented in a heatmap. As the two different bioinformatics pipelines produced $\mathrm{AOB}$ sequences of different lengths, the community dissimilarity between full-length 'assembly' and 'assemblygapped' sequences (i.e. 'assembly' sequences for which the corresponding gap region of the 'gap' pipeline was deleted) were tested on the AOB Illumina MiSeq dataset. Similarly, the significance of the deleted sequence produced in the 'gap' AOA Illumina MiSeq dataset was tested using previously obtained 454 sequences (i.e. 454 full-length sequences vs 454 sequences without the DNA region corresponding to the MiSeq 'gap' and termed '454-gapped'). Pearson correlation and BrayCurtis similarity indices were estimated for each soil sample independently using the cor() and the vegdist() functions from the vegan package on R V3.5.1 [42, 43], respectively, by using the communities produced using the longest read assembly as reference ( $\mathrm{AOB}$ MiSeq 'assembly' and AOA 454 for $\mathrm{AOB}$ and AOA, respectively). Finally, OTU richness of each dataset was estimated using the rarefy function from the vegan package [43] in $\mathrm{R}$, with or without rarefaction to the smallest number of sequences obtained in the different sequencing approaches. The technologies were also compared by phylogenetic comparison using the 454-gapped archaeal amo $A$ sequences (see details of phylogenetic reconstruction below) by estimating the Euclidian distance between the two amoA trees (built with either full- or truncatedlength) using treecompare in the DendroPy library [44]. 


\section{Phylogenetic tree analysis}

Known terrestrial AOB are affiliated to the Nitrosospira genus (including the previously named Nitrosolobus and Nitrosovibrio strains). The amoA and $16 \mathrm{~S}$ rRNA gene sequences of 56 Nitrosospira strains were recovered from NCBI and JGI databases (see Additional file 1: Table S1), were considered as reference sequences and were used to build reference Maximum-Likelihood phylogenetic trees (see below). The AOB amo $A$ reference sequences were also merged with the dereplicated $\mathrm{AOB}$ amo $A$ MiSeq sequences (from the 'assembly' pipeline) and another phylogenetic tree was constructed to assess global diversity in soil. Finally, the dereplicated AOA amoA sequences previously produced on 46 UK soils using 454 were trimmed in the central sequence region (corresponding to the gap region of the MiSeq 'gap' pipeline and resulting in $264 \mathrm{bp}$ ) and a Bayesian phylogenetic tree was constructed to compare to a previously published phylogenetic tree [5].

All sequence datasets were aligned using Mafft [45] and further processed with TrimAl [46] with "- gappyout" flag. For all $a m o A$ datasets, any sequence in which a recombination event was detected using at least 3 of 4 methods (RDP, Bootscan, GENECOV and MaxChi) implemented in RDP4 software [47] was removed after manual curation. Codon saturation was detected by comparing the maximum likelihood distance and the number of differences (Pairwise distance, MEGA 6.06, [48]) for each codon between each pair of sequences. This was statistically assessed using the Xia test implemented in DAMBE [49] and the third codon position was removed.

All AOB phylogenetic trees (both amoA and $16 \mathrm{~S}$ rRNA trees) were constructed using IQ-TREE [50] (with partitioning of the 2 codon positions for the amo $A$ trees) by inferring the best-fit substitution model using ModelFinder [28] and estimating bootstrap supports using the SH-aLRT test [29]. Trees were visualised in FigTree (http://tree.bio.ed.ac.uk/software/figtree/) and phylogenetic clusters were defined based on strong bootstrap values ( $>80 \%$ in most cases). Visual assessment of congruence between the two reference trees ( $a m o A$ vs $16 \mathrm{~S}$ rRNA gene trees) was performed using phylo.io [51] and cluster correspondence was manually assessed based on individual strain correspondence.

For the AOA '454-gapped' Bayesian phylogenetic tree, the best substitution model per codon position estimated using PartitionFinder [52] was $\mathrm{SYM}+\mathrm{G}$ and $\mathrm{GTR}+\mathrm{G}$ for the codon position 1 and 2, respectively, and this partition was used to implement two independent Bayesian relaxed molecular clock phylogenetic analyses in BEAST (Bayesian Evolutionary Analysis Sampling Trees) version 1.8 [53] with $510^{8}$ MCMC, using a Yule speciation prior and an uncorrelated lognormal relaxed clock model. Convergence of the two runs was confirmed using Tracer version 1.5 (tree.bio.ed.ac.uk/ software/tracer/) and maximum clade credibility trees from converged MCMC runs were generated using TreeAnnotator version 1.7 [53] after $50 \%$ of the MCMC steps were removed. Both AOA 454 and AOA 454gapped tree were compared using phylo.io [51] and treecompare in the DendroPy library [44].

\section{AOB putative environmental specialisation}

Environmental factors $(\mathrm{pH}$, nitrogen and carbon contents, C:N ratio, organic matter content (LOI), moisture content and vegetation type) associated with $\mathrm{AOB}$ amo $\mathrm{A}$ sequence composition were identified by canonical correspondence analysis followed by permutation tests performed on relative abundance matrices using the vegan package [43] in R. Relative abundance matrices were built by blasting each $\mathrm{AOB}$ amoA sample sequence obtained from the 50 soils against the bacterial nonredundant database clustered at different cut-offs (usearch id 0.9, 0.95, 0.97 and 1), as performed for the archaeal dataset [54]. As $\mathrm{pH}$ appeared to be the most significant factor for niche specialisation in this $\mathrm{AOB}$ dataset, relative abundance of sequences in the $\mathrm{CEH}$ and Craibstone soil samples affiliating within each phylogenetic cluster, based on the MiSeq 'assembly' pipeline sequencing technology, were represented using a heatmap.

\section{Additional file}

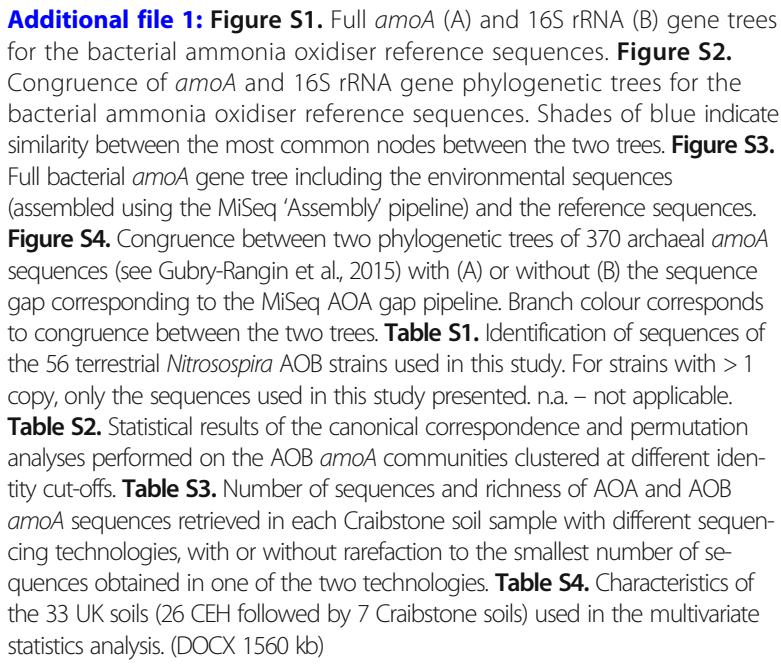

\section{Acknowledgements}

We would like to thank Tony Travis for his support on Linux. We thank Rob Griffiths and Tim Goodall for providing the UK DNA samples and associated contextual data.

Authors' contributions

AA and CGR designed and performed the study, AA and CGR wrote the manuscript, JIP critically revised the manuscript. 


\section{Funding}

AA was supported by a Natural Environmental Research Council grant (NE/ L006286/1) and CGR by a Royal Society University Research Fellowship (UF150571).

\section{Availability of data and materials}

Scripts developed in this work can be found on GitHub (https://github.com/ AigleAxel/amoA_MiSeq_sequencing/). Read data have been submitted to the Sequence Read Archive (SRA) under the accession number PRJNA548755.

\section{Ethics approval and consent to participate}

Not applicable.

\section{Consent for publication}

Not applicable.

\section{Competing interests}

The authors declare that they have no competing interests.

Received: 21 May 2019 Accepted: 6 June 2019

Published online: 04 July 2019

\section{References}

1. Könneke M, Bernhard AE, de la Torre JR, Walker CB, Waterbury JB, Stahl DA. Isolation of an autotrophic ammonia-oxidizing marine archaeon. Nature. 2005:437:543-6.

2. Daims H, Lebedeva EV, Pjevac P, Han P, Herbold C, Albertsen M, Jehmlich N, Palatinszky M, Vierheilig J, Bulaev A, Kirkegaard RH, von Bergen M, Rattei T, Bendinger B, Nielsen PH, Wagner M. Complete nitrification by Nitrospira bacteria. Nature. 2015;528:504-9.

3. van Kessel MA, Speth DR, Albertsen M, Nielsen PH, Op den Camp HJ, Kartal $B$, Jetten MS, Lücker S. Complete nitrification by a single microorganism. Nature. 2015:528:555-9.

4. Gubry-Rangin C, Hai B, Quince C, Engel M, Thomson BC, James P, Schloter M, Griffiths RI, Prosser JI, Nicol GW. Niche specialization of terrestrial archaeal ammonia oxidizers. PNAS. 2011;108:21206-11.

5. Gubry-Rangin C, Kratsch C, Williams TA, McHardy AC, Embley TM, Prosser I, Macqueen DJ. Coupling of diversification and $\mathrm{pH}$ adaptation during the evolution of terrestrial Thaumarchaeota. PNAS. 2015;112:9370-5.

6. Orellana LH, Chee-Sanford JC, Sanford RA, Löffler FE, Konstantinidis KT. Yearround shotgun metagenomes reveal stable microbial communities in agricultural soils and novel Ammonia oxidizers responding to fertilization. Appl Environ Microbiol. 2018;84:e01646-17.

7. Di HJ, Cameron KC, Shen JP, Winefield CS, O'Callaghan M, Bowatte S, He JZ. Nitrification driven by bacteria and not archaea in nitrogen-rich grassland soils. Nat Geosci. 2009:2:621-4.

8. Hink L, Nicol GW, Prosser Jl. Archaea produce lower yields of N2 O than bacteria during aerobic ammonia oxidation in soil. Environ Microbiol. 2017; 19:4829-37.

9. Hink L, Gubry-Rangin C, Nicol GW, Prosser Jl. The consequences of niche and physiological differentiation of archaeal and bacterial ammonia oxidisers for nitrous oxide emissions. ISME J. 2018;12:1084-93.

10. Stephen JR, McCaig AE, Smith Z, Prosser JI, Embley TM. Molecular diversity of soil and marine $16 \mathrm{~S}$ rRNA gene sequences related to $\beta$-subgroup ammonia-oxidizing bacteria. Appl Environ Microbiol. 1996;62:4147-54.

11. Kowalchuk GA, Stephen JR, De Boer W, Prosser Jl, Embley TM, Woldendorp JW. Analysis of ammonia-oxidizing bacteria of the $\beta$-subdivision of the class Proteobacteria in coastal sand dunes by denaturing gradient gel electrophoresis and sequencing of PCR amplified 165 ribosomal DNA fragments. Appl Environ Microbiol. 1997;63:1489-97.

12. Purkhold U, Pommerening-Röser A, Juretschko S, Schmid MC, Koops H-P, Wagner M. Phylogeny of all recognized species of ammonia oxidizers based on comparative $16 \mathrm{~S}$ rRNA and amoA sequence analysis: implications for molecular diversity surveys. Appl Environ Microbiol. 2000;66:5368-82.

13. Purkhold U, Wagner M, Timmermann G, Pommerening-Röser A, Koops HP. 165 rRNA and amoA-based phylogeny of 12 novel betaproteobacterial ammonia-oxidizing isolates: extension of the dataset and proposal of a new lineage within the nitrosomonads. Int J Syst Evol Microbiol. 2003;53:1485-94.
14. Prosser Jl, Head IM, Stein LY. The family Nitrosomonadaceae. In: Rosenberg E, DeLong EF, Lory S, Stackebrandt E, Thompson F, editors. The prokaryotes. Heidelberg: Springer Berlin/Heidelberg; 2014. p. 901-18.

15. Urakawa H, Garcia J, Nielsen J, Le V, Kozlowski J, Stein L, Lim C, Pommerening-Röser A, Martens-Habbena W, Stahl D, Klotz M. Nitrosospira lacus sp. nov., a psychrotolerant, ammonia-oxidizing bacterium from sandy lake sediment. Int J Syst Evol Microbiol. 2015;65(1):242-50.

16. Losos JB. Phylogenetic niche conservatism, phylogenetic signal and the relationship between phylogenetic relatedness and ecological similarity among species. Ecol Lett. 2008;11:995-1003.

17. Wessén E, Nyberg K, Jansson JK, Hallin S. Responses of bacterial and archaeal ammonia oxidizers to soil organic and fertilizer amendments under long-term management. Appl Soil Ecol. 2010;45:193-200.

18. Fierer N, Carney KM, Horner-Devine MC, Megonigal JP. The biogeography of ammonia-oxidizing bacterial communities in soil. Microb Ecol. 2009:58:435-45.

19. Yao H, Campbell CD, Chapman SJ, Freitag TE, Nicol GW, Singh BK. Multifactorial drivers of ammonia oxidizer communities: evidence from a national soil survey. Environ Microbiol. 2013;15:2545-56.

20. Stephen JR, Kowalchuk GA, Bruns MAV, McCaig AE, Phillips CJ, Embley TM, Prosser J. Analysis of beta-subgroup proteobacterial ammonia oxidizer populations in soil by denaturing gradient gel electrophoresis analysis and hierarchical phylogenetic probing. Appl Environ Microbiol. 1998;64:2958-65.

21. Prosser JI, Nicol GW. Archaeal and bacterial ammonia oxidisers in soil: the quest for niche specialisation and differentiation. Trends Microbiol. 2012;20: 523-31.

22. Rotthauwe $\mathrm{JH}$, Witzel KP, Liesack W. The ammonia monooxygenase structural gene amoA as a functional marker: molecular fine-scale analysis of natural ammonia-oxidizing populations. Appl Environ Microbiol. 1997;63: 4704-12.

23. Tourna M, Freitag TE, Nicol GW, Prosser Jl. Growth, activity and temperature responses of ammonia-oxidizing archaea and bacteria in soil microcosms. Environ Microbiol. 2008;10:1357-64.

24. Besser J, Carleton HA, Gerner-Smidt P, Lindsey RL, Trees E. Next-generation sequencing technologies and their application to the study and control of bacterial infections. Clin Microbiol. 2017;24:335-41.

25. Head I, Hiorns WD, Embley TM, McCarthy AJ, Saunders JR. The phylogeny of autotrophic ammonia-oxidizing bacteria as determined by analysis of $16 \mathrm{~S}$ ribosomal RNA gene sequences. J Gen Microbiol. 1993;139:1147-53.

26. Watson SW, Mandel M. Comparison of the morphology and deoxyribonucleic acid composition of 27 strains of nitrifying bacteria. J Bacteriol. 1971;107:563-9.

27. Harms H, Koops HP, Wehrmann H. An ammonia-oxidizing bacterium, Nitrosovibrio tenuis nov. gen. Nov. sp. Arch Microbiol. 1976;108:105-11.

28. Kalyaanamoorthy S, Minh BQ, Wong TKF, von Haeseler A, Jermiin LS. ModelFinder: fast model selection for accurate phylogenetic estimates. Nat Methods. 2017;14:587-9. https://doi.org/10.1038/nmeth.4285.

29. Guindon S, Dufayard J-F, Lefort $V$, Anisimova M, Hordijk W, Gascuel O. New algorithms and methods to estimate maximum-likelihood phylogenies: assessing the performance of PhyML 3.0. Syst Biol. 2010;59:307-21.

30. Norton JM, Alzerreca JJ, Suwa Y, Klotz MG. Diversity of ammonia monooxygenase operon in autotrophic ammonia-oxidizing bacteria. Arch Microbiol. 2002;177:139-49.

31. Klotz MG, Norton JM. Multiple copies of ammonia monooxygenase (amo) operons have evolved under biased AT/GC mutational pressure in ammonia-oxidizing autotrophic bacteria. FEMS Microbiol Lett. 1998; 168:303-11.

32. Khadka R, Clothier L, Wang L, Lim CK, Klotz MG, Dunfield PF. Evolutionary history of copper membrane monooxygenases. Front Microbiol. 2018;9: 2493.

33. Stein LY, Sayavedra-Soto LA, Hommes NG, Arp DJ. Differential regulation of $a m o A$ and $a m o B$ gene copies in Nitrosomonas europaea. FEMS Microbiol Lett. 2000;192:163-8.

34. Koops H-P, Purkhold U, Pommerening-Röser A, Timmermann G, Wagner M. The lithoautotrophic ammonia-oxidizing bacteria. In: Dworkin M, Falkow S, Rosenberg E, Schleifer K-H, Stackebrandt E, editors. The prokaryotes volume 5: Proteobacteria: alpha and Beta subclasses. New York: Springer; 2006. p. 778-811.

35. Griffiths RI, Whiteley AS, O'Donnell AG, Bailey MJ. Rapid method for coextraction of DNA and RNA from natural environments for analysis of ribosomal DNA- and rRNA based microbial community composition. Appl Environ Microbiol. 2000;66:5488-91. 
36. Nicol GW, Leininger S, Schleper C, Prosser Jl. The influence of soil pH on the diversity, abundance and transcriptional activity of ammonia oxidizing archaea and bacteria. Environ Microbiol. 2008;10:2966-78.

37. Krueger F. Trim galore: a wrapper tool around Cutadapt and FastQC to consistently apply quality and adapter trimming to FastQ files. 2015. http:// www.bioinformatics.babraham.ac.uk/projects/trim galore/

38. Callahan BJ, McMurdie PJ, Rosen MJ, Han AW, Johnson AJA, Holmes SP. DADA2: high-resolution sample inference from Illumina amplicon data. Nat Methods. 2016;13:581-3.

39. Zhang J, Kobert K, Flouri T, Stamatakis A. PEAR: a fast and accurate Illumina paired-end reAd mergeR. Bioinformatics. 2014:30:614-20.

40. Edgar RC. Search and clustering orders of magnitude faster than BLAST. Bioinformatics. 2010;26:2460-1.

41. Edgar RC. UNOISE2: improved error-correction for Illumina 165 and ITS amplicon sequencing. BioRxiv. 2016. https://doi.org/10.1101/081257.

42. R Core Team. R: a language and environment for statistical computing: $R$ Found Stat Comput; 2018. https://www.R-project.org/

43. Oksanen J, Blanchet FG, Kindt R. Vegan: community ecology package. R Package Ver. 2013;2:0-10.

44. Sukumaran J, Holder MT. DendroPy: a Python library for phylogenetic computing. Bioinformatics. 2010;26:1569-71.

45. Katoh K, Kuma K, Toh H, Miyata T. MAFFT version 5: improvement in accuracy of multiple sequence alignment. Nucleic Acids Res. 2005;33:511-8.

46. Capella-Gutierrez S, Silla-Martinez JM, Gabaldon T. trimAl: a tool for automated alignment trimming in large-scale phylogenetic analyses. Bioinformatics. 2009;25:1972-3.

47. Martin DP, Lemey P, Lott M, Moulton V, Posada D, Lefeuvre P. RDP3: a flexible and fast computer program for analyzing recombination. Bioinformatics. 2010;26:2462-3.

48. Tamura K, Stecher G, Peterson D, Filipski A, Kumar S. MEGA6: molecular evolutionary genetics analysis version 6.0. Mol Biol Evol. 2013;30:2725-9.

49. Xia X, Xie Z. DAMBE: software package for data analysis in molecular biology and evolution. J Hered. 2017;92:371-3.

50. Nguyen LT, Schmidt HA, von Haeseler A, Minh BQ. IQ-TREE: a fast and effective stochastic algorithm for estimating maximum likelihood phylogenies. Mol Biol Evol. 2015;32:268-74.

51. Robinson O, Dylus D, Dessimoz C. Phylo.lo: interactive viewing and comparison of large phylogenetic trees on the web. Mol Biol Evol. 2016;33: 2163-6.

52. Lanfear R, Calcott B, Ho SYW, Guindon S. PartitionFinder: combined selection of partitioning schemes and substitution models for phylogenetic analyses. Mol Biol Evol. 2012;29:1695-701.

53. Drummond AJ, Suchard MA, Xie D, Rambaut A. Bayesian phylogenetics with BEAUti and the BEAST 1.7. Mol Biol Evol. 2012;29:1969-73.

54. Vico Oton E, Quince C, Nicol GW, Prosser Jl, Gubry-Rangin C. Phylogenetic congruence and ecological coherence in terrestrial Thaumarchaeota. ISME J. 2016;10:85-96.

\section{Publisher's Note}

Springer Nature remains neutral with regard to jurisdictional claims in published maps and institutional affiliations.

Ready to submit your research? Choose BMC and benefit from:

- fast, convenient online submission

- thorough peer review by experienced researchers in your field

- rapid publication on acceptance

- support for research data, including large and complex data types

- gold Open Access which fosters wider collaboration and increased citations

- maximum visibility for your research: over $100 \mathrm{M}$ website views per year

At $\mathrm{BMC}$, research is always in progress.

Learn more biomedcentral.com/submissions 